\title{
Role of the shell thickness in the core transformation of magnetic core(Fe)-shell(Au) nanoparticles
}

\author{
P. Benzo, ${ }^{1}$ S. Combettes, ${ }^{1}$ B. Pecassou, ${ }^{1}$ N. Combe,${ }^{1}$ M. Benoit $\odot,{ }^{1}$ M. Respaud,${ }^{2}$ and M. J. Casanove ${ }^{1, *}$ \\ ${ }^{1}$ CEMES, CNRS UPR 8011 and Université de Toulouse, 29 rue Jeanne Marvig, F-31055 Toulouse, France \\ ${ }^{2}$ LPCNO, Université de Toulouse, INSA, UPS, CNRS, 135 av. de Rangueil, F-31077 Toulouse, France
}

(Received 22 July 2019; published 18 September 2019)

\begin{abstract}
Fe-Au core-shell nanoparticles embedded in an amorphous alumina matrix are synthesized at high temperature using magnetron sputtering. The nanoparticles display a single-crystal Fe core covered by a crystalline Au shell epitaxially grown on the different core facets. The morphology of the grown nanoparticles is analyzed by transmission electron microscopy, while their structural details are resolved at the atomic scale using probecorrected high-angle annular dark-field scanning transmission microscopy. Two different epitaxial relationships at the $\mathrm{Au} / \mathrm{Fe}$ interface are observed at low Au coverage, whereas only one type of interface orientation remains when the shell thickness exceeds 3 monolayers (MLs). This leads to a drastic Fe core transformation from a Wulff shaped crystal towards a nanocube. This core transformation drives a surface reconstruction resulting in a combination of open and close-packed strain free facets, offering different opportunities for molecule binding. In addition, our experiments show that the magnetic properties of the Fe core are preserved by the Au shell and that the $10 \mathrm{~nm}$ size of the grown nanoparticles favors a superparamagnetic behavior, suitable for biomedical applications.
\end{abstract}

DOI: 10.1103/PhysRevMaterials.3.096001

\section{INTRODUCTION}

Combining a magnetic core with a biocompatible shell, Fe-Au core-shell nanoparticles (NPs) are highly promising candidates for biomedical applications, among which magnetic resonance imaging, magnetically driven drug delivery, or hyperthermia [1-4]. Indeed, pure iron presents better magnetic properties than its oxides, for instance a higher saturation magnetization, and the chemically inert gold shell provides efficient protection against the easy oxidation and the toxicity of the iron core. Nonetheless, the gold shell also offers a suitable platform for bioconjugation $[5,6]$.

Different strategies have thus been developed to synthesize $\mathrm{Fe}-\mathrm{Au}$ NPs with this desired chemical distribution, particular attention being paid to the quality of the gold coverage. The core-shell distribution should be favored owing to the much lower surface energy of gold compared to iron [7] and the low miscibility of these elements at room temperature [8]. This tendency is clearly confirmed by calculations [9-11]. However, synthesis through wet chemistry methods proved to be highly challenging in this system, in particular for preventing the iron core from oxidation [12], and better results are reported using different routes. Thus, Fe@ Au core-shell NPs have been successfully synthesized from alloy targets using laser irradiation [2], laser ablation in liquid [10,13,14], solid-state dewetting of bimetallic films [15], or magnetron sputtering [16].

Beyond the core-shell chemical order, the properties of $\mathrm{Fe} @ \mathrm{Au}$ NPs also rely on the crystalline quality of both domains. Indeed, structural disorder decreases the magnetization in crystalline $\mathrm{Fe}$ [17]. Moreover, in case of polycrystalline

\footnotetext{
*casanove@cemes.fr
}

Au shell, the grain boundaries will presumably influence the shell porosity to Fe oxidizing agent. The structural features of the NPs in turn influence their morphology and thus the nature of the exposed surface facets, which affects the possibility of molecule binding for biological applications.

In a previous paper [16] we succeeded in growing wellfaceted Fe@Au nanocrystals using thermally activated deposition by magnetron sputtering. The synthesized NPs displayed an original morphology consisting of a cubic Fe core surrounded by six Au truncated pyramids. The stability of this geometry displaying a centered core, unusual in low miscible systems [18], was attributed to the large surface energy contribution compared to the small elastic [due to a low misfit at the $\mathrm{Au}(100) / \mathrm{Fe}(100)$ interface] and interface energies $[19,20]$.

In this paper we investigate the role of the shell thickness on the nanoparticle morphology, special attention being paid to the $\mathrm{Au}$ shell/Fe core interface structure. The core-shell nanoparticles have been grown by magnetron sputtering according to the same procedure as previously reported. This procedure indeed proved well adapted to the synthesis of NPs with low size dispersity and in the $10 \mathrm{~nm}$ range, suited for biomedical applications. We describe the evolution of the core morphology with increasing Au deposited volume, from the equilibrium shape described by Wulff [21] observed in pure Fe NPs to the already reported cubic Fe core. Contrary to the study by Kamp et al. [13] who observed the evolution of a rounded Fe core towards a cubic core upon in situ heating of spherical Fe@Au NPs grown at room temperature, our study compares the morphology of NPs which are grown at a same temperature. This temperature is sufficiently high to promote both a highly crystalline quality and a VolmerWeber (3D) growth mode in our setting. Owing to these 
experimental conditions, the reported evolution with Au:Fe ratio is analyzed in terms of interface competition. Most interestingly, the morphology resulting from the thickening of the $\mathrm{Au}$ shell combines interesting properties for biomedical applications, i.e., a superparamagnetic behavior, thanks to the well-preserved properties of the Fe core, and neat open and close-packed Au free surfaces for bioconjugation.

\section{EXPERIMENTAL DETAILS}

The bimetallic FeAu NPs were grown from two elemental targets in a ultrahigh vacuum sputtering device. A $5 \mathrm{~nm}$ thick amorphous $\mathrm{Al}_{2} \mathrm{O}_{3}$ buffer layer was first deposited at room temperature onto a freshly cleaved $\mathrm{NaCl}$ crystalline substrate. The substrate temperature was then increased to $600{ }^{\circ} \mathrm{C}$, and the two metallic layers were deposited sequentially, Fe film first. The temperature was then slowly decreased towards room temperature at which the grown nanoparticles were covered by a $5 \mathrm{~nm}$ thick amorphous $\mathrm{Al}_{2} \mathrm{O}_{3}$ capping layer. Due to the synthesis conditions, the elemental ratio at the nanoparticle scale cannot be ascertained. Indeed, only the nominal deposited thickness of the two metals is known and the chemical composition of the nanoparticles, which are formed at high temperature, will depend on different factors among which surface diffusion of the two metals on the alumina sublayer, interface energies, or preferential reevaporation of one of the metals. For this reason, the growth conditions being identical in all the samples, we only refer to the nominal deposited thickness of each metal and the samples will be called $\mathrm{Fe}_{x} \mathrm{Au}_{y}, x$ and $y$ being the nominal deposited thickness of each metal, in $\mathrm{nm}$.

Plan-view specimens of the deposited films were prepared for transmission electron microscopy (TEM) experiments and scanning transmission electron microscopy (STEM) analyses, after dissolution of the $\mathrm{NaCl}$ substrate in deionized water. The experiments were conducted in a Cs-corrected FEI Tecnai F20 microscope for TEM analyses and in a probe-corrected JEOL cold FEG ARM200F microscope for atomically resolved high-angle annular dark-field (HAADF) STEM experiments. Both microscopes were operated at $200 \mathrm{kV}$.

A fused silica substrate was used to prepare the samples for magnetometry measurements. The sequence of deposition $\mathrm{Al}_{2} \mathrm{O}_{3}(5 \mathrm{~nm}) / \mathrm{Fe}(x \mathrm{~nm}) / \mathrm{Au}(y \mathrm{~nm}) / \mathrm{Al}_{2} \mathrm{O}_{3}(5 \mathrm{~nm})$ was reproduced twice in order to increase the signal to noise ratio, the two layers of NPs being thus separated by a 10 $\mathrm{nm}$ thick $\mathrm{Al}_{2} \mathrm{O}_{3}$ layer. The magnetic properties of the NPs were measured using vibrating sample magnetometry (VSM) in a PPMS Quantum Design system. Magnetization data were recorded along the sample plane.

\section{RESULTS AND DISCUSSION}

\section{A. Core morphology versus shell thickness}

Figure 1 presents low magnification TEM images and associated size distribution histograms of three different samples grown with $x=1 \mathrm{~nm}$ and varying $y$, namely (a) pure $\mathrm{Fe}$ $\left(\mathrm{Fe}_{1} \mathrm{Au}_{0}\right)$; (b) $y=0.5 \mathrm{~nm}\left(\mathrm{Fe}_{1} \mathrm{Au}_{0.5}\right)$; (c) $y=1 \mathrm{~nm}\left(\mathrm{Fe}_{1} \mathrm{Au}_{1}\right)$. All the images present well dispersed NPs characteristic of a Volmer-Weber growth mode on the amorphous alumina
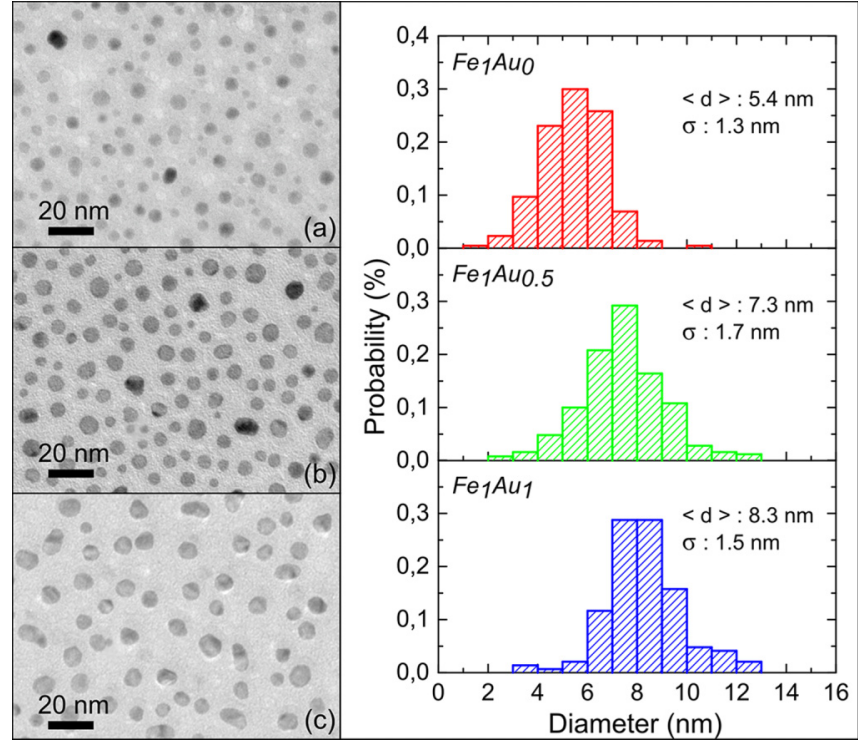

FIG. 1. TEM micrographs and corresponding size histograms of $\mathrm{Fe}-\mathrm{Au}$ NPs grown using different values of Au nominal deposited thickness. The mean diameter and standard deviation of each histogram are reported in the graphs captions.

substrate. Besides, the three samples display a narrow size distribution whose characteristics are reported in Table I, together with the density of NPs in each sample (details on TEM images analysis are given in the Supplemental Material [22]). The increase of the mean NP diameter $\langle d\rangle$ with increasing Au deposited thickness, is consistent with a diffusion of $\mathrm{Au}$ atoms towards the $\mathrm{Fe}$ and $\mathrm{Fe}-\mathrm{Au} \mathrm{NPs}$, at least partly, a desorption of $\mathrm{Au}$ atoms remaining possible. Besides, we did not observe in these samples the formation of pure $\mathrm{Au}$ clusters. Interestingly, the NPs density in sample $\mathrm{Fe}_{1} \mathrm{Au}_{1}$ is noticeably lower than the density in the $\mathrm{Fe}_{1} \mathrm{Au}_{0.5}$ sample. As observed in the corresponding histogram, this decrease is mainly due to the absence of the smaller NPs presumably resulting from either Ostwald ripening or coalescence favored by the increase of the NPs volume.

Whatever their composition, the bimetallic nanoparticles present a core-shell Fe-Au chemical distribution, as better observed in the high-angle annular dark-field scanning transmission electron microscopy (HAADF-STEM) images displayed in Fig. 2. Owing to the large difference in the atomic number of the two metals, the Au shell displays a much brighter contrast than the center of the NPs which contains an important proportion of $\mathrm{Fe}$ atoms. In addition to this

TABLE I. Size (mean diameter $\langle d\rangle$ and standard deviation $\sigma$ ) and spatial (density of NPs) distributions of the nanoparticles in the samples reported in Fig. 1. Note that the density of the NPs was calculated on much larger regions than the ones displayed in Fig. 1.

\begin{tabular}{lccc}
\hline \hline Sample & $\begin{array}{c}\langle d\rangle \\
(\mathrm{nm})\end{array}$ & $\begin{array}{c}\sigma \\
(\mathrm{nm})\end{array}$ & $\begin{array}{c}\text { Density } \\
\left(\mathrm{NPs} / \mathrm{cm}^{2}\right)\end{array}$ \\
\hline $\mathrm{Fe}_{1} \mathrm{Au}_{0}$ & 5.4 & 1.3 & $6.7 \times 10^{11}$ \\
$\mathrm{Fe}_{1} \mathrm{Au}_{0.5}$ & 7.3 & 1.7 & $7.7 \times 10^{11}$ \\
$\mathrm{Fe}_{1} \mathrm{Au}_{1}$ & 8.3 & 1.5 & $4.5 \times 10^{11}$ \\
\hline \hline
\end{tabular}



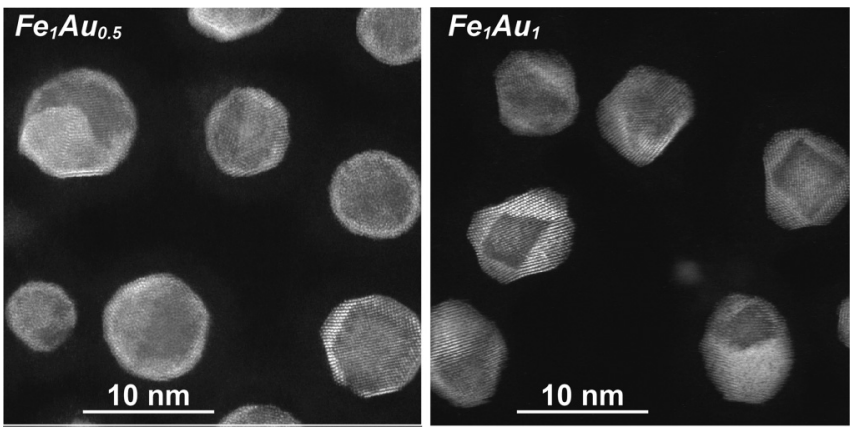

FIG. 2. HAADF-STEM images of the two samples $\mathrm{Fe}_{1} \mathrm{Au}_{0.5}$ and $\mathrm{Fe}_{1} \mathrm{Au}_{1}$ giving evidence for a core-shell morphology. The Au rich regions appear brighter, thanks to the sensitivity of HAADF images to the atomic number of the elements. Comparison between these two images also reveals important differences between the two samples and in particular a fully different NP core morphology.

core-shell chemical order, the displayed images reveal a significant morphological difference between the two samples. In the $\mathrm{Fe}_{1} \mathrm{Au}_{0.5}$ sample, most of the NP cores appear bounded by 6 to 8 main facets of approximately equal length and the thin $\mathrm{Au}$ shell reproduces the same morphology. In contrast, a large proportion of the NPs observed in the $\mathrm{Fe}_{1} \mathrm{Au}_{1} \mathrm{HAADF}-\mathrm{STEM}$ image exhibit a square-shaped core bounded by four main facets and the $\mathrm{Au}$ shell seems to form faceted islands on each of the square facets. Larger regions of these samples are presented in the additional HAADF-STEM images displayed in the Supplemental Material [22].

Except for this $Z$ contrast typical of HAADF-STEM images, the two different domains, core and shell, are also distinguished by their crystalline structure, body-centered cubic (bcc) for Fe and face-centered cubic (fcc) for Au. This structural difference can be observed in the NPs presented in Fig. 3. Both NPs are here observed along a $\langle 100\rangle$ direction of the Fe bcc lattice, an orientation particularly suitable to observe neat $\mathrm{Au} / \mathrm{Fe}$ interfaces. In the $\mathrm{Fe}_{1} \mathrm{Au}_{0.5}$ sample, the Fe core appears bounded by $\{110\}_{\mathrm{Fe}}$ and $\{100\}_{\mathrm{Fe}}$ families of lattice planes. In contrast, sample $\mathrm{Fe}_{1} \mathrm{Au}_{1}$ displays a very typical morphology consisting of a cubic Fe core bounded by $\{100\}_{\mathrm{Fe}}$ lattice planes, and surrounded by six Au truncated pyramids. This morphology was already fully detailed in a previous paper [16].

\section{B. Structure of the $\mathrm{Au} / \mathrm{Fe}$ interface}

The above results give evidence for the formation of essentially two different heterophase interfaces between fcc Au and bcc Fe, depending on the Fe core facet $\{100\}_{\mathrm{Fe}}$ or $\{110\}_{\mathrm{Fe}}$

Let us first consider the $\{100\}_{\mathrm{Fe}}$ facets, which coincide with the cube faces in cubic Fe core. As previously emphasized [16], the Au shell can grow easily on $\{100\}_{\mathrm{Fe}}$ crystal plane according to the $\mathrm{Au}\{100\}\langle 100\rangle / / \mathrm{Fe}\{100\}\langle 110\rangle$ epitaxial relationship, also known as the Bain orientation relationship (see Fig. 4 left). Indeed, owing to the unit cell parameters of the two metals, $a_{\mathrm{Fe}}=0.28665 \mathrm{~nm}$ and $a_{\mathrm{Au}}=0.40784 \mathrm{~nm}$ [8], the lattice misfit at this interface is very low and writes $f=\left(a_{\mathrm{Au}}-a_{\mathrm{Fe}} \sqrt{2}\right) / a_{\mathrm{Fe}} \sqrt{2}=0.6 \%$.

The second type of commonly observed core facet is the bcc highest-density plane $\{110\}_{\mathrm{Fe}}$. In this case, the interface

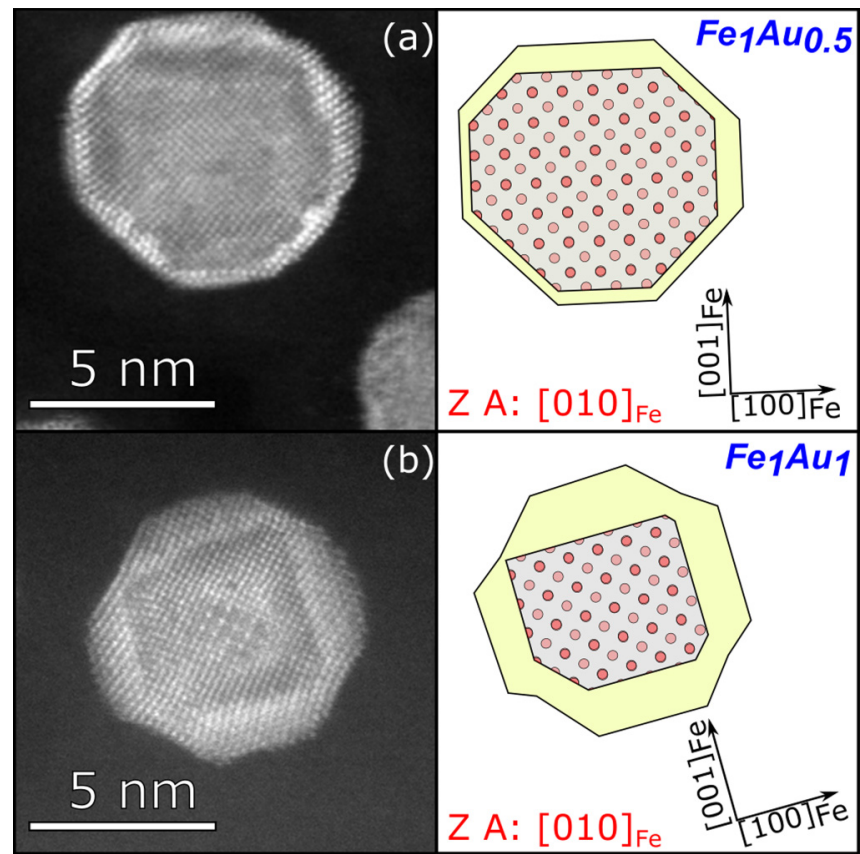

FIG. 3. Atomically resolved HAADF-STEM images of Fe-Au core-shell NPs observed along a $[010]_{\mathrm{Fe}}$ axis and associated sketches: (a) $\mathrm{Fe}_{1} \mathrm{Au}_{0.5}$ sample; (b) $\mathrm{Fe}_{1} \mathrm{Au}_{1}$ sample. Note that in the NP displayed in (b), a moiré pattern originating from the superposition of the Fe core with the top and bottom Au pyramids, is observed in the NP center. A scheme of the atomic structure of the Fe core is given in the sketches to indicate the observed orientations of the core facets.

plane in the Au lattice is expected to be the $\{111\}_{\mathrm{Au}}$ plane, i.e., the fcc highest-density plane. Two orientations are then possible depending if the $\mathrm{Au}$ most densely packed atomic row, $\langle 110\rangle_{\mathrm{Au}}$, aligns parallel to either $\langle 111\rangle_{\mathrm{Fe}}$ or $\langle 001\rangle_{\mathrm{Fe}}$ directions. The corresponding relationships at the interface are respectively known as the Kurdjumov-Sachs (KS) and the Nishiyama-Wasserman (NW) orientations. In our samples, the dimensions of the core facets and thus the interface areas are very small so that precise orientation cannot be ascertained

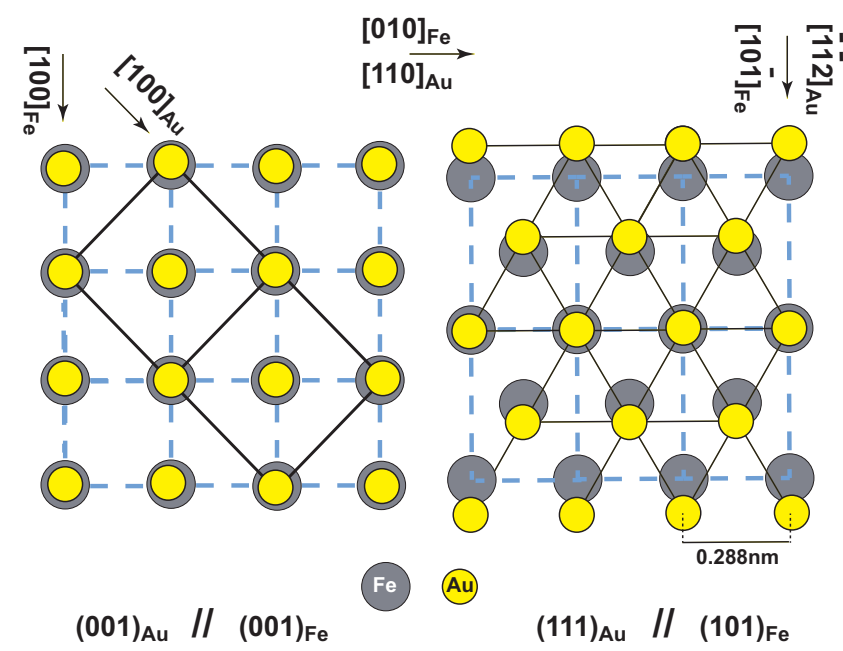

FIG. 4. Plan view of the Au/Fe interface. Left: Bain orientation relationship. Right: Nishiyama-Wasserman orientation relationship. 
from TEM analyses. It is however admitted in thin films that the preferred orientation depends on the ratio of the nearest-neighbor distances in both crystals [23]. In the $\mathrm{Au} / \mathrm{Fe}$ system, the nearest-neighbor distance is $0.28838 \mathrm{~nm}$ in $\mathrm{Au}$ and $0.2482 \mathrm{~nm}$ in $\mathrm{Fe}$, leading to a ratio $r=1.162$, close to the value of 1.155 for which the NW orientation is preferred. This orientation writes $\mathrm{Au}\{111\}\langle 110\rangle / / \mathrm{Fe}\{110\}\langle 100\rangle$, or equivalently $\mathrm{Au}\{111\}\langle 112\rangle / / \mathrm{Fe}\{110\}\langle 110\rangle$, see Fig. 4 right. It was recently confirmed in Fe films grown on $\{111\}_{\mathrm{Au}}$ [24]. It should be noted that this orientation relationship leads to an anisotropic lattice misfit [25]. Indeed, there are three identical $\langle 110\rangle$ rows in the $\{111\}_{\mathrm{Au}}$ interface plane and only one can be parallel to $\langle 100\rangle_{\mathrm{Fe}}$ in a same crystal. Along this direction, the misfit will be the same as the one given for the Bain relationship, i.e., 0.6\%. Along the perpendicular direction, i.e., parallel to $\langle 110\rangle_{\mathrm{Fe}}$, the misfit $f=\left(a_{\mathrm{Au}} \sqrt{3 / 2}-\right.$ $\left.a_{\mathrm{Fe}} \sqrt{2}\right) / a_{\mathrm{Fe}} \sqrt{2}$ is as high as $23 \%$. The Au layer will then be heavily compressed if it grows coherently over a $\{110\}_{\mathrm{Fe}}$ core facet. In thin films, as both fcc $\{111\}_{\mathrm{Au}}$ and bcc $\{110\}_{\mathrm{Fe}}$ are atomically flat, the lattice misfit is expected to relax through the introduction of misfit dislocations with in-plane Burgers vectors. In our case, owing to the small dimensions of the interfaces, the formation of such dislocations may be impeded and the Au layer may accommodate the misfit through other types of structural defects, as ledges or tilt boundaries [26].

\section{Core transformation mechanism}

The core morphology evolution as a function of the $\mathrm{Au}$ shell thickness can be derived from these different observations. As shown in Fig. 1(a), pure Fe NPs synthesized using the same growth conditions, display a roughly rounded shape. This morphology is consistent with the model of Wulff polyhedron [21] calculated by Saito for bcc crystals [27]. In this model, when only first and second near-neighbor interactions are considered in the calculation, the polyhedron is a rhombic dodecahedron truncated by $\{100\}$ facets, thus exposing mainly $\{110\}$ and $\{100\}$ facets. When higher order terms of interactions are taken into account, the edges and corners of the polyhedron are rounded due to the occurrence of differently oriented very small facets. This effect seems to be particularly active for Fe nanocrystals [27]. The deposition of a thin $\mathrm{Au}$ shell, as in sample $\mathrm{Fe}_{1} \mathrm{Au}_{0.5}$, favors a polyhedral core morphology close to the truncated rhombic dodecahedron with neat edges and interface facets parallel to $\{110\}_{\mathrm{Fe}}$ and $\{100\}_{\mathrm{Fe}}$, as in Fig. 3(a). The core morphology then evolves towards a cubic shape, increasing the Au:Fe volume ratio in the NPs clearly promoting the growth of $\{100\}_{\mathrm{Fe}}$ facets to the detriment of the small $\{110\}_{\mathrm{Fe}}$ facets. This implies a diffusion of Fe atoms from $\{110\}_{\mathrm{Fe}}$ to $\{100\}_{\mathrm{Fe}}$ facets. The high growth temperature used in our synthesis facilitates this diffusion.

The observed evolution of the Fe core with the $\mathrm{Au}$ deposited volume can be interpreted as resulting from an energy minimization mechanism. Although it is difficult to quantify the different contributions, we still can compare the energy cost of the different morphologies. In case of a cubic core, only one type of interface is concerned, namely the $\mathrm{Au}\{100\}\langle 100\rangle / / \mathrm{Fe}\{100\}\langle 110\rangle$ epitaxial interface, and it has a low interfacial energy [19]. In contrast, reliable estimates of the interfacial energy at the $\{111\}_{\mathrm{Au}} /\{110\}_{\mathrm{Fe}}$ interface are not available, to the best of our knowledge. Nevertheless, one can reasonably expect that the energy cost of combined interfacial, elastic, and surface contributions will be much higher than in the previous growth direction. Indeed, either the Au shell will grow coherently under a high compressive strain, thus increasing the elastic energy contribution, or it will grow semicoherently or incoherently, with introduction of structural defects increasing the interfacial energy. As observed on the HAADF-STEM images, the Au shell grown on $\{110\}_{\mathrm{Fe}}$ facets seems to remain flat and parallel to $\{111\}_{\mathrm{Au}}$, i.e., the $\mathrm{Au}$ facet with lower surface energy. However, this facet is most probably strained, at least partially, contrary to the $\{111\}_{\mathrm{Au}}$ pyramids lateral facets, and this may be the reason for the limited height of the Au overgrowth (a few MLs) on $\{110\}_{\mathrm{Fe}}$ facets. The shear modulus of bulk $\mathrm{Au}$ being much lower than the one of bulk Fe (27 vs $82 \mathrm{GPa}$ ), the strain energy should be preferentially accommodated by the $\mathrm{Au}$ overgrowth. Minimization of the NPs energy should thus promote the development of larger $\{100\}_{\mathrm{Fe}}$ facets with increasing $\mathrm{Au}$ volume to the detriment of the $\{110\}_{\mathrm{Fe}}$ facets.

The NPs shape evolves accordingly. Beginning by a thin uniform shell over the Wulff-shaped Fe NPs, we observe in the $\mathrm{Fe}_{1} \mathrm{Au}_{0.5}$ sample the rising of Au pyramids on $\{100\}_{\mathrm{Fe}}$ core facets, while the $\{110\}_{\mathrm{Fe}}$ facets are coated by a 2-3 MLs thick $\mathrm{Au}$ shell. With increasing the Au:Fe ratio, pyramids grow on all the faces of the now cube-shaped core. As previously shown, the slight compressive strain accommodated by the $\mathrm{Au}$ overgrowth on a Fe cube facet is soon relaxed through the large area of free surface resulting from the pyramidal shape [16]. This pyramidal shape also reduces the surface energy in the NPs owing to the numerous $\{111\}_{\mathrm{Au}}$ close-packed lateral facets. The $\mathrm{Au}$ pyramids observed in the $\mathrm{Fe}_{1} \mathrm{Au}_{1}$ sample are topped by open $\{100\}_{\mathrm{Au}}$ facets, thus offering different possibilities for molecule binding and increasing the interest of this typical morphology for biomedical applications.

\section{Magnetic properties of the Fe-Au core-shell NPs}

The magnetic properties of an assembly of $\mathrm{Fe}_{1} \mathrm{Au}_{1}$ NPs were investigated and compared with the properties of an assembly of Fe NPs (sample $\mathrm{Fe}_{1} \mathrm{Au}_{0}$ ). To increase the magnetic signal, samples with two layers of NPs separated by a thick alumina layer $(10 \mathrm{~nm})$ have been prepared. Figure 5 presents isothermal $M(H)$ hysteresis loops measured at 2.5 and $300 \mathrm{~K}$, the magnetic field being applied along the substrate. Paramagnetic and diamagnetic contributions from magnetic impurities and/or substrate have been subtracted from the raw data. For the sake of comparison, the magnetic measurements have been normalized according to the low temperature and high magnetic field saturation values. As expected, these NPs are ferromagnetic. For each system, the hysteresis loops do not depend on the magnetic history, after either a cooling without a magnetic field (ZFC) or with a field cooling (FC) procedure at a magnetic field of $6 \mathrm{~T}$ from 390 and down to $2.5 \mathrm{~K}$. It is an indirect confirmation of the metallic character of the Fe NPs. Indeed, the presence of some Fe oxide layer would induce an exchange bias and shifted hysteresis loops. Interestingly, the $\mathrm{Au}$ deposit leads to an improved squareness. At $2.5 \mathrm{~K}$ the remanent/saturation magnetizations ratio increases up to 0.6 for $\mathrm{Fe}_{1} \mathrm{Au}_{1}$ in comparison to 0.26 for $\mathrm{Fe}_{1} \mathrm{Au}_{0}$ NPs. The 

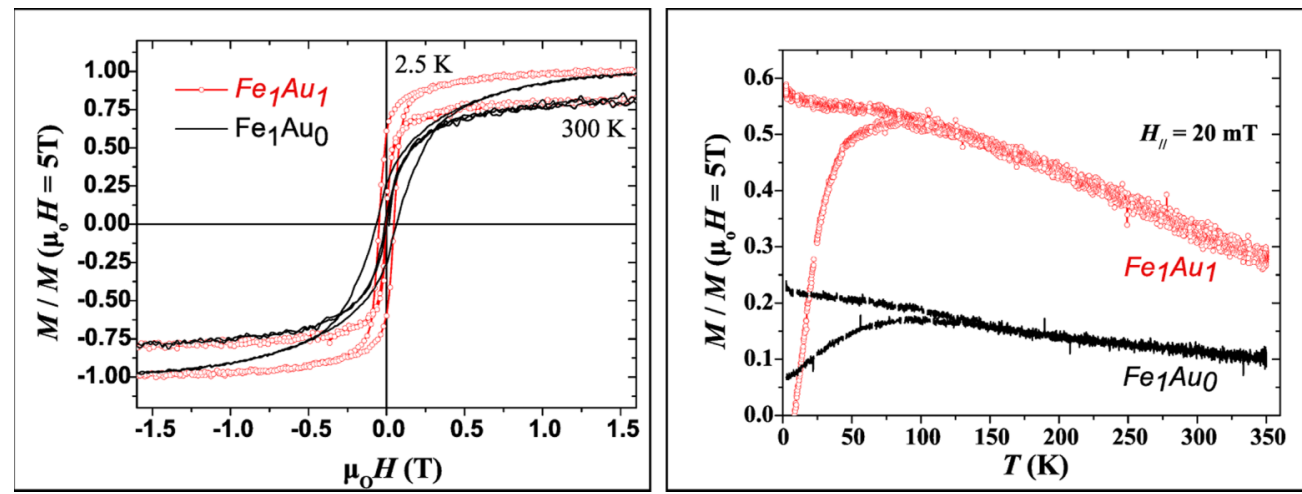

FIG. 5. Magnetic properties of $\mathrm{Fe}_{1} \mathrm{Au}_{1}$ and $\mathrm{Fe}_{1} \mathrm{Au}_{0} \mathrm{NPs}$ : (a) Isothermal $M(H)$ hysteresis loops recorded at 2.5 and $300 \mathrm{~K}$ after a $\mathrm{FC}$ under $6 \mathrm{~T}$ from $390 \mathrm{~K}$. (b) ZFC/FC magnetization measurements for a $20 \mathrm{mT}$ in-plane applied field.

coercive fields are 47.5 and $64 \mathrm{mT}$ for $\mathrm{Fe}_{1} \mathrm{Au}_{1}$ and $\mathrm{Fe}_{1} \mathrm{Au}_{0}$ $\mathrm{NPs}$, respectively. Upon increasing the temperature the coercive field reduces and superparamagnetic behaviors are observed above $100 \mathrm{~K}$ in both cases. This behavior is confirmed through the measure of the magnetization versus temperature according to a zero-field cooling (ZFC)-field cooling (FC) procedure for an in-plane applied magnetic field of $20 \mathrm{mT}$ [see Fig. 5(b)]. These measurements have been normalized using the same procedure as for the hysteresis loops. They evidence a superparamagnetic behavior of the assembly of NPs. The blocking temperatures, defined as the maximum of the $\mathrm{ZFC}$ curve are about 90 and $100 \mathrm{~K}$ for $\mathrm{Fe}_{1} \mathrm{Au}_{1}$ and $\mathrm{Fe}_{1} \mathrm{Au}_{0}$ respectively. These values cannot be discussed in a simple way, since the applied magnetic field is of the same order than the coercive field (47.5 and $65 \mathrm{mT}$ for $\mathrm{Fe}_{1} \mathrm{Au}_{1}$ and $\mathrm{Fe}_{1} \mathrm{Au}_{0}$, respectively). Indeed, the blocking temperature decreases with the applied magnetic field divided by the coercive field [28]. Note that the magnetization of the $\mathrm{Fe}_{1} \mathrm{Au}_{1} \mathrm{NPs}$ is higher than the one for $\mathrm{Fe}_{1} \mathrm{Au}_{0} \mathrm{NPs}$, in agreement with the hysteresis shape. This set of experimental data allows us to conclude that the Au layer promotes a better definition of the easy magnetization axis in the NPs plane. This is linked to the morphology and crystalline properties. The well faceted and oriented $\mathrm{Fe}$ shape for $\mathrm{Fe}_{1} \mathrm{Au}_{1}$ NPs may reinforce the in-plane easy axis. However, discussing the influence of the Au shell on the magnetic anisotropy energy clearly deserves a deeper magnetic study.

\section{CONCLUSION}

In summary, we succeeded in growing $\mathrm{Fe}-\mathrm{Au}$ core-shell nanoparticles with narrow size distribution in the $10 \mathrm{~nm}$ range using a UHV magnetron sputtering device. The use of high temperature of $600^{\circ} \mathrm{C}$ during the metal deposition favors the growth of crystalline nanoparticles and limits the number of grain boundaries, preferential oxygen diffusion paths towards the NP core. A first consequence of the crystalline character of the nanoparticles is the occurrence of well defined coreshell interfaces. Comparing the structural and morphological features of NPs with various $\mathrm{Au}: \mathrm{Fe}$ volume ratio, we gave evidence for an evolution of the type of Fe core facets with the $\mathrm{Au}$ shell thickness. More particularly, our results show the progressive vanishing of the $\{110\}_{\mathrm{Fe}}$ core facets in favor of $\{100\}_{\mathrm{Fe}}$ facets with increasing Au deposited thickness. The core morphology thus evolves from a roughly rounded shape to a truncated rhombic dodecahedron (Wulff equilibrium shape) to reach a cubic shape at high Au coverage. The NP morphology evolves accordingly, from a rounded or Wulff shape for small Au shell thickness towards a highly faceted shape formed of a cubic Fe core surrounded by six truncated Au pyramids. The origin of such morphological evolution was attributed to the difference in interfacial and elastic energies at the core-shell interface depending on the interface orientation relationship, Bain or Nishiyama-Wasserman. Lowering the energy cost of the NW Au/Fe interface is seen as the driving force for the $\mathrm{Fe}$ core reconstruction in a nearly perfect cube. Most interestingly, the NPs with cubic cores not only display well preserved magnetic properties, but also expose a combination of strain free open and close-packed Au facets, which make them very attractive for bioconjugation. These findings should open new perspectives for the design of core-shell NPs for biomedical applications.

\section{ACKNOWLEDGMENTS}

This study has been (partially) supported through the grant NEXT No. ANR-10-LABX-0037 in the framework of the "Programme des Investissements d'Avenir". We gratefully acknowledge for their assistance, Lucien Datas and Teresa Hungria with the ARM200F at Centre de MicroCaracterisation Raymond Castaing in Toulouse and Sebastien Pinaud for the magnetization measurements at LPCNO.
[1] S. Kayal and R. V. Ramanujan, J. Nanosci. Nanotechnol. 10, 5527 (2010).

[2] J. Zhang, M. Post, T. Veres, Z. J. Jakubek, J. Guan, D. Wang, F. Normandin, Y. Deslandes, and B. Simard, J. Phys. Chem. B 110, 7122 (2006).
[3] Z. Ban, Y. A. Barnakov, F. Li, V. O. Golub, and C. J. O'Connor, J. Mater. Chem. 15, 4660 (2005).

[4] M. Chen, S. Yamamuro, D. Farrell, and S. A. Majetich, J. Appl. Phys. 93, 7551 (2003). 
[5] Q. Sun, A. K. Kandalam, Q. Wang, P. Jena, Y. Kawazoe, and M. Marquez, Phys. Rev. B 73, 134409 (2006).

[6] D. Huber, Small 1, 482 (2005).

[7] W. Tyson and W. Miller, Surf. Sci. 62, 267 (1977).

[8] H. Okamoto, T. Massalski, L. Swartzendruber, and P. Beck, Bull. Alloy Phase Diagrams 5, 592 (1984).

[9] S. Hong and T. S. Rahman, Phys. Chem. Chem. Phys. 17, 28177 (2015).

[10] P. Wagener, J. Jakobi, C. Rehbock, V. S. K. Chakravadhanula, C. Thede, U. Wiedwald, M. Bartsch, L. Kienle, and S. Barcikowski, Sci. Rep. 6, 23352 (2016).

[11] F. Calvo, N. Combe, J. Morillo, and M. Benoit, J. Phys. Chem. C 121, 4680 (2017).

[12] S.-J. Cho, A. M. Shahin, G. J. Long, J. E. Davies, K. Liu, F. Grandjean, and S. M. Kauzlarich, Chem. Mater. 18, 960 (2006).

[13] M. Kamp, A. Tymoczko, U. Schürmann, J. Jakobi, C. Rehbock, K. Räzke, S. Barcikowski, and L. Kienle, Crystal Growth Design 18, 5434 (2018).

[14] A. Tymoczko, M. Kamp, O. Prymak, C. Rehbock, J. Jakobi, U. Schürmann, L. Kienle, and S. Barcikowski, Nanoscale 10, 16434 (2018).

[15] D. Amram and E. Rabkin, ACS Nano 8, 10687 (2014).
[16] C. Langlois, P. Benzo, R. Arenal, M. Benoit, J. Nicolai, N. Combe, A. Ponchet, and M. J. Casanove, Nano Lett. 15, 5075 (2015).

[17] Y. Kakehashi, T. Uchida, and M. Yu, Phys. Rev. B 56, 8807 (1997).

[18] D. Bochicchio and R. Ferrando, Phys. Rev. B 87, 165435 (2013).

[19] M. Benoit, C. Langlois, N. Combe, H. Tang, and M.-J. Casanove, Phys. Rev. B 86, 075460 (2012).

[20] M. Benoit, N. Combe, A. Ponchet, J. Morillo, and M.-J. Casanove, Phys. Rev. B 90, 165437 (2014).

[21] G. Wulff, Z. Kristallographie 34, 449 (1901).

[22] See Supplemental Material at http://link.aps.org/supplemental/ 10.1103/PhysRevMaterials.3.096001 for more details on TEM/STEM image analysis and additional images.

[23] E. Bauer and J. H. van der Merwe, Phys. Rev. B 33, 3657 (1986).

[24] H. F. Jurca, A. Damian, C. Gougaud, D. Thiaudière, R. Cortès, F. Maroun, and P. Allongue, J. Phys. Chem. C 120, 16080 (2016).

[25] H. Elmers and U. Gradmann, Surf. Sci. 304, 201 (1994).

[26] D. Amram and E. Rabkin, Acta Mater. 61, 4113 (2013).

[27] Y. Saito, J. Cryst. Growth 53, 273 (1981).

[28] W. T. Coffey and Y. P. Kalmykov, J. Appl. Phys. 112, 121301 (2012). 